\title{
Examining commute routes: applications of GIS and GPS technology
}

\author{
Hannah M. Badland • Mitch J. Duncan • \\ Melody Oliver $\cdot$ J. Scott Duncan $\cdot$ Suzanne Mavoa
}

Received: 26 October 2009/Accepted: 16 February 2010/Published online: 20 March 2010

(C) The Japanese Society for Hygiene 2010

\begin{abstract}
Objective The application of geographic information systems (GIS) to describe commute route elements is commonplace, yet the accuracy of GIS-estimated commute routes is not clear. This study compared GIS-estimated commute routes against actual routes traveled as measured using global positioning systems (GPS) to examine differences in urban form surrounding travel routes across different buffer sizes and travel modes.

Methods Thirty-seven adults from Auckland, New Zealand participated in the study between January and March 2008. Participants wore GPS units and completed a travel $\log$ for 7 days. GPS data were integrated into a GIS database to ascertain commute routes.

Results Overall, 29 commute journeys were appropriately captured by GPS. Levels of agreement between actual and shortest commute routes were dependent on the buffer size selected, built environment variable examined, and travel mode.

Conclusions Despite technical difficulties, GPS assessment of routes traveled is recommended to provide an
\end{abstract}

H. M. Badland $(\bowtie) \cdot$ M. Oliver · J. S. Duncan

Centre for Physical Activity and Nutrition Research,

Faculty of Health and Environmental Sciences,

Auckland University of Technology,

Private Bag 92006, Auckland, New Zealand

e-mail: hannah.badland@aut.ac.nz

\section{J. Duncan}

Centre for Social Science Research,

CQUniversity, Rockhampton, Australia

S. Mavoa

Centre for Social and Health Outcomes Research

and Evaluation, Massey University, Auckland, New Zealand accurate assessment of commute journey urban form elements.

Keywords Adults · Environment .

Transport-related physical activity · Travel · Work

\section{Introduction}

In recent years, a substantial amount of research has examined the relationship between the built environment and physical activity engagement. Measures to assess urban form have become increasingly sophisticated as this field has developed, shifting from a reliance on broad environmental classifications, self-report measures, and total physical activity accumulation to the use of more confined settings, objective measurement tools, and specific activity domains. Transport-related physical activity (TPA), which incorporates walking and cycling for travel purposes, including accessing public transport (PT), has attracted attention from public health and planning disciplines as it can provide a valuable source of physical activity accumulation while reducing automobile infrastructure provision and congestion [1]. The commute route environment [2] and associated travel distances [3] have been identified as important correlates of TPA engagement, and there is scope to compare these variables with objective measurement tools, such as geographical information systems (GIS) and global positioning systems (GPS).

GIS is regarded as the gold standard for objectively assessing built environments. For example, a recent study investigated the agreement between self-reported proximity to a park with GIS-determined crow-fly distance [4]. Agreement was poor between the two measures $(62 \%$, kappa $=0.095)$, and the authors concluded that 
destination-specific data should be taken objectively wherever possible. Furthermore, GIS is becoming a progressively popular measure to objectively assess the built environment-TPA relationship as it allows complex physical variables, such as mixed land use, residential density, and street connectivity, to be examined with relative ease. Research suggests that these three urban form measures influence TPA [5]. A further strength of GIS is that it can be used to model commute routes at the individual level based on the shortest street network distance using the closest facility function. To date, several studies have applied GIS to simulate commute routes and examine built environment features that adults [6,7] and children [8] travel through, comparing these environments by travel modes. One study delimited the sample to adults commuting less than $5 \mathrm{~km}$ to access their workplace and compared street connectivity, mixed land use, and residential density using GIS-estimated measures. The results showed those who commuted through the most connected street networks, as determined by the shortest GIS-estimated street network commute, were sixfold as likely to travel by TPA modes when compared with adults traveling through the least connected environments [6].

Duncan and Mummery [9] recently compared GPS- and GIS-determined trip lengths and traffic volume exposure in children walking or cycling short distances (mean $1.08 \mathrm{~km}$, range $4.36 \mathrm{~km}$ ) to travel to and from school. Travel distances were the same for the two approaches, but the GISestimated commute route crossed over significantly more busy streets than the GPS-measured network. This information is useful; however, it is also important to determine if simulated GIS commute routes are comparable to actual commute routes by different travel modes, for important urban form correlates beyond travel distance and busy streets, and for longer journeys, such as the commute to work. The application of GPS units to help answer these questions is ideal; these are non-obtrusive, objective monitoring devices that spatially track where an individual moves. However, the integration of GPS and GIS in TPA research is relatively new.

The pilot study reported here builds upon the emerging evidence base by comparing GPS- and GIS-estimated commute routes, built environment commute route variables, and travel modes for adults commuting between a residential and occupational setting and seeks to investigate whether the GIS-estimated shortest commute route can be appropriately used to estimate the types of environment that people travel through. These findings will determine in part whether it is appropriate to apply a GIS-estimated commute network, or if simultaneous actual route measures are required for travel research in the adult population. This has important implications for research methods in this field and provides a clearer understanding of how built environments are associated with travel behaviors in the adult population.

\section{Methods}

Study subjects

University employees were recruited between January and March 2008 (summer) from Auckland, New Zealand. The host institution ethics committee provided study approval (07/218). The reader is referred to Oliver et al. [10] for a detailed description of the methodology.

Travel mode

Participants completed a 7-day self-report travel log for all work-related journeys. Trackstick II (Telespial Systems, Marina Del Rey, CA) GPS units were worn for 7 days during waking hours except for water-based activities. Participants remained stationary for 1 min outside prior to commencing each journey to allow for GPS satellite acquisition. Data from the travel log and GPS were matched for each journey. Travel modes were collapsed into private vehicle travel and TPA/PT prior to analysis.

\section{Urban design measures}

Participants' residential and occupational addresses were geo-coded. The closest facility function on ArcView v9.2 software was used to model the participants' shortest street network commute route between their home and work addresses (hereafter referred to as the 'shortest' commute). GPS data were exported to ArcView v9.2 to identify the actual commute path between home and work for each commute trip (hereafter referred to as the 'actual' commute). Buffer distances from the road median were developed at 25,100 , and $250 \mathrm{~m}$ radii along the actual and shortest networks for each trip.

Consistent with current health geography research [11], calculations of residential density, land use mix, and street connectivity were performed for these buffer sizes. Briefly, residential density was estimated using mesh-block data on the normal resident population from the New Zealand 2006 census [12]. A mesh-block is defined as a geographic census unit of approximately 100 households constructed by Statistics New Zealand [12]. Residential land area was obtained from the land use and zoning data provided by the local government agencies. Residential density was calculated by dividing the number of dwellings by the residential land area for each mesh-block. The mesh-blocks intersecting each buffer were used to calculate a weighted average of the residential population density based on the land area. 
Table 1 Mean and mean difference (95\% limits of agreement) between all travel modes and commute route buffers for built environment variables

\begin{tabular}{|c|c|c|c|c|c|c|}
\hline & \multicolumn{2}{|l|}{$25 \mathrm{~m}$} & \multicolumn{2}{|l|}{$100 \mathrm{~m}$} & \multicolumn{2}{|l|}{$250 \mathrm{~m}$} \\
\hline & $\chi$ & $\begin{array}{l}\text { Mean difference } \\
\text { (95\% limits of } \\
\text { agreement) }\end{array}$ & $\chi$ & $\begin{array}{l}\text { Mean difference } \\
\text { (95\% limits of } \\
\text { agreement) }\end{array}$ & $\chi$ & $\begin{array}{l}\text { Mean difference } \\
\text { (95\% limits of } \\
\text { agreement) }\end{array}$ \\
\hline \multicolumn{7}{|l|}{ Land use mix } \\
\hline Actual and shortest route agreement & 0.03 & $(-0.13$ to 0.19$)$ & 0.03 & $(-0.14$ to 0.20$)$ & 0.00 & $(-0.14$ to 0.14$)$ \\
\hline Private vehicle & 0.03 & $(-0.14$ to 0.19$)$ & 0.04 & $(-0.15$ to 0.22$)$ & 0.01 & $(-0.14$ to 0.17$)$ \\
\hline $\mathrm{TPA} / \mathrm{PT}$ & 0.03 & $(-0.11$ to 0.17$)$ & 0.00 & $(-0.12$ to 0.12$)$ & -0.02 & $(-0.09$ to 0.04$)$ \\
\hline \multicolumn{7}{|l|}{ Residential density (population) } \\
\hline Actual and shortest route agreement & -107 & $(-529$ to 315$)$ & -30 & $(-163$ to 103$)$ & -12 & $(-69$ to 45$)$ \\
\hline Private vehicle & -63 & $(-354$ to 229$)$ & -14 & $(-91$ to 63$)$ & -5 & $(-35$ to 25$)$ \\
\hline TPA/PT & -203 & $(-805$ to 399$)$ & -64 & $(-265$ to 137$)$ & -29 & $(-115$ to 58$)$ \\
\hline \multicolumn{7}{|l|}{ Street connectivity } \\
\hline Actual and shortest route agreement & -0.01 & $(-0.14$ to 0.12$)$ & 0.02 & $(-0.18$ to 0.22$)$ & 0.00 & $(-0.12$ to 0.12$)$ \\
\hline Private vehicle & -0.01 & $(-0.11$ to 0.09$)$ & 0.03 & $(-0.11$ to 0.17$)$ & 0.01 & $(-0.10$ to 0.11$)$ \\
\hline $\mathrm{TPA} / \mathrm{PT}$ & 0.00 & $(-0.20$ to 0.19$)$ & -0.01 & $(-0.31$ to 0.28$)$ & -0.03 & $(-0.16$ to 0.11$)$ \\
\hline
\end{tabular}

$T P A$ Transport-related physical activity, $P T$ public transport

The land use and zoning data were used to categorize land uses into commercial, residential, industrial, open space, and 'other' within each mesh-block. The land use mix assigned to each commute journey was calculated using an entropy index [13], where 0 indicated homogeneity of land use, and a value closer to 1 specified greater heterogeneity of land uses.

Street connectivity was estimated by calculating intersection density. Street intersections with three or more unique intersecting streets were extracted from road network data. Mesh-block boundaries were typically defined by street centerlines. Therefore, to ensure that street intersections coincidental with mesh-block boundaries were included, intersection density was calculated as the number of intersections per square kilometer within $20 \mathrm{~m}$ of each mesh-block boundary. Values for each mesh-block were between 0 and 1 , where a score closer to 1 indicated higher street connectivity. The treatment of these urban form variables was consistent that reported in the literature [11].

\section{Statistical analysis}

Bland and Altman methodology was used to examine differences between the actual and shortest commute routes for urban design measures at different buffer sizes and travel modes. The procedure incorporated a pair-wise comparison showing the relative bias (mean difference) and the mean difference $95 \%$ limits of agreement $( \pm 1.96$ $\mathrm{SD}$ of the mean difference) between the shortest (test) and the actual (reference) commute routes.

\section{Results}

The overall sample comprised 37 participants, resulting in a possible 370 journeys. However, only 29 separate journeys from work to residence, or vice versa, were captured by GPS, with the modal share being $69 \%$ by private vehicle, $3 \%$ by PT, and $28 \%$ by TPA modes. Fifteen participants provided these latter data (trip range 1-5). All further results pertain to the 29 journeys recorded by the GPS units. The $95 \%$ limits of agreement between the actual and shortest commute routes remained relatively constant when land use mix was examined (see Table 1). As the buffer size increased, so did agreement between the actual and shortest commute route measures for residential density. The level of agreement between the actual and the shortest commute route for street connectivity varied according to buffer size.

Actual and shortest commute routes were also compared by travel modes across the built environmental variables. Higher levels of agreement were evident for TPA/PT modes than for private vehicle travel in terms of the land use mix ratio. The levels of agreement narrowed as buffer sizes increased for residential density.

\section{Discussion}

The purpose of this paper was to investigate whether the GIS-estimated shortest commute route could be appropriately used to estimate the types of environment that people travel through. Our results illustrate that levels of 
agreement between the actual and the shortest commute route were dependent on the buffer size selected, built environment variable examined, and travel mode addressed. Larger buffer sizes yielded a higher level of agreement between the shortest and actual commute route, and this relationship held true for when the travel modes were separated.

Our findings suggest that the mean difference limits of agreement for land use mix and street connectivity ratios were too wide to recommend the application of the shortest commute route in travel research. Residential density, however, appears to have adequate agreement at buffer distances of 100 and $250 \mathrm{~m}$ when compared to values reported in previous studies [3]. When we compared our findings with previous results, the limits of agreement are within the smallest quartile range for residential density at $100 \mathrm{~m}$ (266 residents this study, 295 residents previous study), but they are higher for land use mix ratio at $100 \mathrm{~m}$ ( 0.40 this study, 0.10 previous study) and street connectivity ratio at $250 \mathrm{~m}(0.24$ this study, 0.04 previous study) [3].

In addition, previous research has demonstrated that GPS technology when combined with GIS databases can accurately measure commute routes, especially when compared to self-reported journeys [14]. Accordingly, GPS technology will likely continue to improve, including becoming better integrated with readily available technology, such as mobile phones. Improvements may also be seen in GPS signal acquisition and increased ease of integration with existing software programs, such as GIS and online mapping tools (e.g., Google Maps).

There are several limitations to this pilot study, with the most obvious being the non-representativeness of the group and the small sample of 29 trips. Failure to locate a GPS signal during the initialization period resulted in significant data loss, as GPS requires a clear line of sight to the sky to operate accurately. Accordingly, potential reasons for not acquiring satellites during the initialization period include: participants not remaining stationary outside for the specified time period when they left buildings, the initialization signal may have been blocked by buildings or natural features (e.g., verandahs, mountains), or the signal may have been bounced off buildings before being received by the satellite (i.e., urban canyon effect). Strategies to overcome these issues should be considered in future GPS research. Additionally, participants traveled to a common workplace, which may have reduced the variability in urban form measures, and each journey was treated as an independent observation; therefore, potential exists for inflating the risk of a type I error. Despite these issues, we recommend objectively assessing the commute route where possible to provide a more accurate depiction of urban form encountered during the journey. The application of differing buffer zones should be considered for specific urban form attributes when examining commute routes.

Acknowledgments This study was funded by a Building Research Capacity in the Social Sciences Grant (Grant Number: PR57855) and a New Zealand National Heart Foundation Small Project Grant (Grant Number: 1299). HMB and MO are supported by National Heart Foundation of New Zealand Research Fellowships. No conflicts of interest exist for this manuscript.

\section{References}

1. Sallis JF. Measuring physical activity environments. Am J Prev Med. 2009;36:S86-92.

2. Lee C, Vernez Moudon A. The 3D's + R: quantifying land use and urban form correlates of walking. Trans Res Part D Transp Environ. 2006;11:204-15.

3. Badland HM, Schofield GM, Schluter PJ. Objectively measured commute distance: Associations with actual travel modes and perceptions to place of work or study in Auckland, New Zealand. J Phys Act Health. 2007;4:80-6.

4. Macintyre S, Macdonald L, Ellaway A. Lack of agreement between measured and self-reported distance from public green parks in Glasgow, Scotland. Int J Behav Nutrit Phys Act. 2008;5:26.

5. Saelens BE, Handy SL. Built environment correlates of walking: A review. Med Sci Sport Exerc. 2008;40:S550-66.

6. Badland HM, Schofield GM, Garrett N. Travel behavior and objectively measured urban design variables: Associations for adults traveling to work. Health Place. 2008;14:85-95.

7. Rodriquez D, Joo J. The relationship between non-motorized mode choice and the local physical environment. Trans Res Part D Transp Environ. 2004;9:151-73.

8. Timperio A, et al. Personal, family, social, and environmental correlates of active commuting to school. Am J Prev Med. 2006;30:45-51.

9. Duncan MJ, Mummery WK. GIS or GPS? A comparison of the two methods for assessing route taken during active transport. Am J Prev Med. 2007;33:51-3.

10. Oliver M, et al. GPS, GIS, and accelerometry: Methodological issues in the assessment of location and intensity of travel behaviors. J Phys Act Health. 2010;7:102-8.

11. Badland HM, et al. Understanding the Relationship between Activity and Neighbourhoods (URBAN) Study: Research design and methodology. BMC Public Health. 2009;9:224.

12. Statistics New Zealand. Census 2006. Statistics New Zealand: Wellington; 2007.

13. D'Sousa E, et al. Twin cities walking study. In: Forsyth A, editor. Environment and physical activity: GIS protocols. Version 3.1. Minneapolis: Metropolitan Design Center; 2006.

14. Wolf J, et al. Accuracy issues with route choice data collection by using Global Positioning System. Transport Research Record. 1999;1660:64-79. 